\title{
Modified Lees-Edwards Boundary conditions and viscous contact for numerical simulations of particles in a shear flow
}

\author{
Nicolas Verdon ${ }^{\mathrm{a}}$, Aline Lefebvre-Lepot ${ }^{\mathrm{b}}$, Patrice Laure ${ }^{\mathrm{a}, \mathrm{c} *}$ and Laurent Lobry ${ }^{\mathrm{d}}$ \\ ${ }^{a}$ Laboratoire J.A. Dieudonné, UMR CNRS 7351, Université de Nice, Parc Valrose, 06108 Nice Cedex \\ 02, France; ${ }^{b}$ Centre de Mathématiques Appliquées (CMAP), Ecole Polytechnique, Route de Saclay, \\ 91128 Palaiseau, France; ${ }^{c}$ Mines ParisTech, CEMEF, UMR CNRS 7635, Sophia Antipolis, 06560 \\ Valbonne, France; ${ }^{d}$ Laboratoire de Physique de la Matière Condensée (LPMC), Université de \\ Nice-Sophia Antipolis, Parc Valrose, 06108 Nice Cedex 02, France
}

\begin{abstract}
We present a way to handle contacts between rigid particles in shear flow. The influence of such a modeling is shown by studying an example with 13 particles in 3D. Studying a concentrated suspension in 2D, we demonstrate that contact modelling as well as choice of boundary conditions influences the macroscopic properties of the suspension.

Cet article présente une modélisation du contact entre particules solides dans un écoulement cisaillé. On montre ici l'importance du modèle de contact sur un cas d'étude 3D comportant 13 particules. Une analyse plus détaillée sur une suspension concentrée 2D montre l'influence des conditions aux limites et de la description du contact sur le déplacement des particules et les paramétres rhéologiques des suspensions.
\end{abstract}

Keywords: suspension; contact; viscous contact model; fluid-structure interaction; immersed domain method

Mots-clés: suspension; contact; modèle de contact visqueux; interaction fluide-structure

\section{Introduction}

In the field of materials forming, as well as in many other industrial fields, determining the rheological behaviour of dense suspensions remains of great importance. Lots of different computational methods can be used to handle such fluid-structure interactions but in this work, we propose to study the behaviour of solid particles in a shear flow with the immersed domain method. First introduced in the late 1990s by Glowinski, Pan, Helsa, and Joseph (1999), these methods encounter an increasing success in fluid-structure or multiphase problems. In this way, the computations are made in a static domain with an Eulerian approach (each phase being described by a characteristic or level set function).

Before considering a large amount of particles, we explain the methodology on the description of contact for a few particles in a shear flow. To avoid particle overlapping during displacement of particles, contact models which handle both inelastic collision and viscous contact are compared. The later model was introduced by Maury (2007) and has been intensively studied by Lefebvre $(2007,2009)$ for granular applications.

*Corresponding author. Email: patrice.laure@unice.fr 
We then focused our analysis on the choice of the boundary conditions for describing dense suspensions in a representative way. We present in detail the way of extending the computational domain and we focus on the description of sliding biperiodic conditions of Lees-Edwards (Beaume, 2008; Hwang, Hulsen, \& Meijer, 2004; Lees \& Edwards, 1972).

In the last part of this article, we present some representative results that confirm the different choices we have done in this study. First, the importance of the contact modelling will be pointed out with a 3D example of 13 particles in a shear flow. Finally, some examples of suspensions will also demonstrate the rheological modifications due to the choice of boundary conditions.

\section{Numerical model}

In this part, we briefly describe the main features of the numerical model that has been used for this study. The importance of the contact modelling is therefore explained at the end of the section.

\subsection{The immersed domain approach}

The immersed domain method is achieved by splitting the computational domain $\Omega$ into two subdomains $\Omega_{\mathrm{f}}(t)$ and $\Omega_{\mathrm{s}}(t)$, respectively, for the fluid and solid parts (see Figure 1). Note that, these two subdomains evolve in time (depending on the position of the particles). In the case of multiple particles, the solid domain is the union of domains corresponding to each particle, namely $\Omega_{\mathrm{s}}(t)=\bigcup_{i}^{N} \Omega_{\mathrm{s}_{i}}(t)$ for $N$ particles. We restrict our study to monodisperse suspensions and all the particles are spheres (or circles) of radius $a$.

The interface $\Gamma_{\mathrm{s}}(t)$ between the two phases is described by the zero isosurface of a signed distance function:

$$
\alpha(\mathbf{x}, t)>0 \quad \text { if } \quad \mathbf{x} \in \Omega_{\mathrm{s}}(t) \quad \text { or } \quad<0 \quad \text { if } \mathbf{x} \notin \Omega_{\mathrm{s}}(t)
$$

which allows to define a "smooth" characteristic function:

$$
\square(\mathbf{x}, t)= \begin{cases}=1 & \text { if } \alpha(\mathbf{x}, t)>e \\ =\frac{\alpha(\mathbf{x}, t)}{e} & \text { if } 0<\alpha(\mathbf{x}, t)<e \\ =0 & \text { if } \alpha(\mathbf{x}, t)<0\end{cases}
$$

where $e$ the mixing thickness depends on the mesh size around the interface. In addition, a global viscosity $\eta$ is defined thanks to a mixing relation:

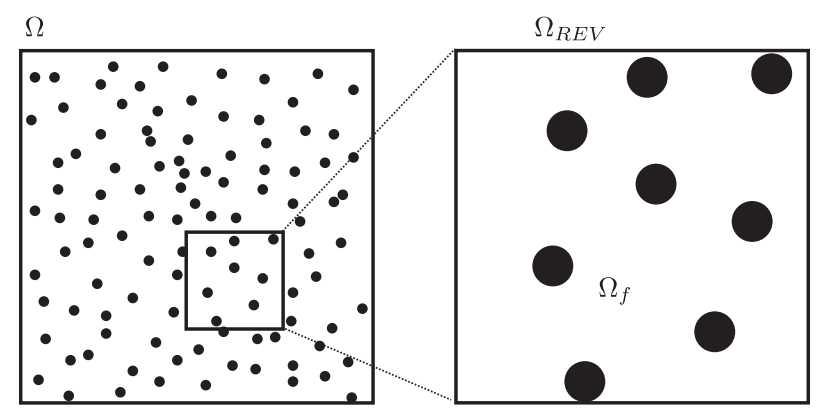

Figure 1. Computational domain $\Omega$ and fluid domain $\Omega_{\mathrm{f}}$ with spherical particles; homogenisation is made on representative elementary volume $\Omega_{\mathrm{REV}}$. 


$$
\eta=\llbracket \eta_{\mathrm{s}}+(1-\llbracket) \eta_{\mathrm{f}}
$$

where $\eta_{\mathrm{f}}$ is the fluid viscosity and $\eta_{\mathrm{s}} \simeq r \eta_{\mathrm{f}}$ is the solid viscosity (or penalty factor) usually taken much bigger than $\eta_{\mathrm{f}}\left(r \approx 10^{3}\right)$.

\subsection{Governing equations}

Neglecting inertia and gravity, the fluid-solid problem can be written with the following set of equations:

$$
\left\{\begin{array}{l}
\nabla \cdot \sigma=0 ; \nabla \cdot \mathbf{u}=0 \\
{[[\mathbf{u}]]_{\Gamma_{\mathrm{s}}}=0 ;[[\sigma \cdot \mathbf{n}]]_{\Gamma_{\mathrm{s}}}=0 \text { on } \Gamma_{\mathrm{s}}} \\
\mathbf{u}=\mathbf{u}_{\Gamma} \text { on the external boundary } \Gamma
\end{array}\right.
$$

where $\mathbf{u}$ is the fluid velocity, $\dot{\epsilon}(\mathbf{u})$ is the rate of strain tensor, $\sigma$ is the stress tensor $\left(\sigma=2 \eta_{\mathrm{f}} \dot{\epsilon}(\mathbf{u})-p \mathbf{I}\right.$ for Newtonian fluid, $\mathbf{E}$ being the identity matrix), $p$ is the pressure and $\eta_{\mathrm{f}}$ is the fluid viscosity (the symbol $[[f]]_{\Gamma_{\mathrm{s}}}$ means the jump of scalar $f$ across the interface $\Gamma_{\mathrm{s}}$ ). Patankar, Singh, Joseph, Glowinski, and Pan (2000) have proposed to extend the above Stokes equation to the solid domain thanks to a Lagrange multiplier by using the rigidity condition $\dot{\epsilon}(\mathbf{u})=0$ on $\Omega_{\mathrm{s}}$. In this way, the motion in solid domain $\Omega_{\mathrm{s}}$ corresponds to a fluid motion with an additional stress field. Indeed, if $\lambda$ is the corresponding Lagrange multiplier, this is equivalent to take the stress tensor $\sigma$ inside the solid domain of the form

$$
\sigma=2 \eta_{\mathrm{s}} \dot{\epsilon}(\mathbf{u})-p \mathbf{I}+\dot{\epsilon}(\lambda)
$$

\subsection{Weak formulation of the FSI problem}

Equation (5) allows us to write the following weak formulation over the whole computational domain $\Omega$, where Dirichlet boundary conditions are imposed:

Find $(\mathbf{u}, p, \lambda)$ such that $\forall(\mathbf{v}, q, \mu) \in \mathcal{H}_{0}^{1}(\Omega) \times \mathcal{L}_{0}^{2}(\Omega) \times \mathcal{H}^{1}\left(\Omega_{\mathrm{s}}\right)$ :

$$
\left\{\begin{array}{l}
0=\int_{\Omega} 2 \eta \dot{\epsilon}(\mathbf{u}): \dot{\epsilon}(\mathbf{v}) d \Omega-\int_{\Omega} p \nabla \cdot \mathbf{v} d \Omega+\int_{\Omega_{\mathrm{s}}} \dot{\epsilon}(\lambda): \dot{\epsilon}(\mathbf{v}) d \Omega \\
0=\int_{\Omega} q \nabla \cdot \mathbf{u} d \Omega \\
0=\int_{\Omega_{\mathrm{s}}} \dot{\epsilon}(\mu): \dot{\epsilon}(\mathbf{u}) d \Omega
\end{array}\right.
$$

where $\mathcal{H}_{0}^{1}(\Omega)$ is the classical Sobolev space satisfying the Dirichlet boundary condition on $\Gamma$. This formulation corresponds to an augmented Lagrangian function where $\lambda$ is the Lagrange multiplier and $\eta_{\mathrm{s}}$ is the penalty factor. This equation is solved using an Uzawa algorithm (Laure, Beaume, Basset, Silva, \& Coupez, 2007).

\subsection{Contact modelling}

The particles displacement is then achieved by using the velocity of the fluid, solution of Equation (6) and a Lagrangian approach. Namely the position $\mathbf{X}_{i}^{n+1}$ of particle $i$ at time $t^{n+1}=t^{n}+\Delta t$ is computed with the second-order Adams-Bashfort scheme:

$$
\mathbf{X}_{i}^{n+1}=\mathbf{X}_{i}^{n}+\frac{\Delta t}{2}\left[3 \mathbf{V}_{i}^{n}-\mathbf{V}_{i}^{n-1}\right]
$$


where $\mathbf{V}_{i}^{n}$ is the velocity of particle $i$ at time $t^{n} . \mathbf{V}_{i}^{n}$ is computed from the velocity field obtained by solving Equation (6). It can be shown that, theoretically, using the solutions to this continuous Stokes problem shall allow to avoid overlappings between the particles. Indeed, the lubrication force acting on neighbouring particles is sufficient to prevent the distances to go to zero in finite time. However, since this force becomes singular when the distance goes to zero, we have to cope with very stiff systems and it has been observed that numerical overlappings can occur, due to space and time discretisations. In order to avoid this non-physical behaviour, we used the viscous contact model that has been introduced by Maury (2007) and Lefebvre (2009), which allows to avoid overlappings and takes the lubrication forces into account.

This model can be seen as a predictor-corrector model based on the action of the lubrication force. The predicted velocity, $\mathbf{V}_{i}^{n, *}=\mathbf{u}\left(\mathbf{X}_{i}^{n}, t^{n}\right)$ will be corrected in a way that it satisfies the non-overlapping condition, namely by solving a least-square problem:

$$
\frac{1}{2}\left|\mathbf{V}^{n}-\mathbf{V}^{n, *}\right|^{2}=\min _{\mathbf{V} \in K\left(\mathbf{X}^{n}, \gamma_{i j}^{n}\right)} \frac{1}{2}\left|\mathbf{V}-\mathbf{V}^{n, *}\right|^{2}
$$

where $K$ is the space of admissible velocity defined by

$$
K\left(\mathbf{X}^{n}, \gamma_{i j}^{n}\right)=\left\{\mathbf{V} \mid \begin{array}{l}
\widetilde{D}_{i j}^{n+1}(\mathbf{V}) \geq 0 \quad \forall \quad i, j \\
\widetilde{D}_{i j}^{n+1}(\mathbf{V}) \leq 0 \text { if } \gamma_{i j}^{n}<0
\end{array}\right\}
$$

where $\widetilde{D}_{i j}^{n+1}$ is the estimation at first order and at time $n+1$ of the distance between particle $i$ and $j$ :

$$
\widetilde{D}_{i j}^{n+1}(\mathbf{V})=D_{i j}^{n}+\frac{3 \Delta t}{2}\left(\mathbf{V}_{j}-\mathbf{V}_{i}\right) \cdot \mathbf{e}_{i j}^{n}-\frac{\Delta t}{2}\left(\mathbf{V}_{j}^{n-1}-\mathbf{V}_{i}^{n-1}\right) \cdot \mathbf{e}_{i j}^{n}
$$

where $\mathbf{e}_{i j}$ is the unitary vector connecting the centres of particle $i$ and $j$. The constraints in (9) are put in the form of the two inequality in order to introduce two Karush-Kuhn-Tucker multipliers. Then, the problems (8) and (9) are put in a classical saddle-point form by introducing two Lagrange multipliers $\lambda_{i j}^{ \pm}$for each couple $i j$ of particles which satisfy the Kuhn-Tucker conditions (Kuhn \& Tucker, 1951). These two Lagrange multipliers can be linked to lubrication forces and give the correction for velocities. Their values are obtained using again an Uzawa procedure (Verdon, Lefebvre-Lepot, Lobry, \& Laure, 2010; Verdon, Beaume, Lefebvre-Lepot, Lobry, \& Laure, submitted).

With this approach, the contact between particles is then described by the new variable $\gamma_{i j}$ which can be seen as a microscopic distance between particles $i$ and $j$ :

$$
\gamma_{i j}^{n}=\left\{\begin{array}{l}
<0 \text { if there is contact between particles } i \text { and } j \\
=0 \text { else }
\end{array}\right.
$$

The evolution of $\gamma_{i j}^{n}$ is given by the following equation

$$
\frac{d \gamma_{i j}}{d t}=-\frac{1}{a^{2}} \lambda_{i j} \quad \text { with } \quad \lambda_{i j}=\lambda_{i j}^{+}-\lambda_{i j}^{-}
$$

where $a$ is the radius of particles. 
Note that the inelastic collisions model can easily be implemented from this model by imposing $\gamma_{i j} \equiv 0$, that is to say $\lambda_{i j}>0$.

\section{Description of boundary conditions}

When increasing the concentration of solid particles in a suspension, we notice that the choice of the boundary conditions becomes of great importance. Indeed, the number of contacts between particles is also increased and hence the boundary conditions (BC) can affect the rheology of the suspension. In this section, we present different choices that can be made and we focus on a Lee-Edwards biperiodic conditions which extends the usual periodic boundary conditions in the shear direction.

\subsection{Representative elementary volume}

In the numerical study of suspension, it is important to work on a suitable representative elementary volume (REV) in order to get suitable macroscopic values. Ideally, we would like to be able to know the behaviour of suspensions in a large computational domain $\Omega$ with boundaries far enough from the domain of interest $\Omega_{\mathrm{REV}}$, as depicted in Figure 1 .

Unfortunately, this kind of approach is nowadays very computationally expensive, especially for $3 \mathrm{D}$ simulations. That is why we have to deal with the boundary conditions in order to have a suitable representative domain. For low concentrated suspension, it is common to work with a computational domain equal to the REV but if the concentration increases, it is no more representative. Indeed, in $\Omega_{\mathrm{REV}}$ the influence of the particles from the domain $\Omega \backslash \Omega_{\mathrm{REV}}$ are taken into account whereas in a smaller domain such that $\Omega \equiv \Omega_{\mathrm{REV}}$, the particles near the boundary do not see particles from outside and their computed velocities will be affected by imposed boundary conditions.

\subsection{Extension of the computational domain and sliding biperiodic boundary conditions}

In the computational domain $\Omega$, the velocities on the upper and lower boundaries are hence imposed in order to get the desired shear rate $\dot{\gamma}$, namely $U$ is equal to $\pm \dot{\gamma} H / 2$, where $H$ is the height of the computational domain. In addition all velocity components normal to the boundary are equal to zero in order to get a perfect Couette flow. Finally, the computation of macroscopic variables are made on an unitary square (or cubic) $\Omega_{\mathrm{REV}}$. Initially for dilute suspensions, the computational domain $\Omega$ is equal to representative elementary volume $\Omega_{\mathrm{REV}}$ as dipicted in Figure 2(a). In this case, we impose a "weak" periodic condition on the horizontal directions. That means that each particle that goes outside the domain through vertical walls re-enters in the domain from the other side. However, the velocities may be incorrectly estimated, due to the conditions of a null normal velocity and to the fact that we do not take into account the particles that are outside of $\Omega_{\mathrm{REV}}$ but near its boundary. Indeed, although the motion of particles is periodic, their computed velocities are not. That is why we proposed to extend the computational domain in the $x$-direction as depicted in Figure 2(b). In this extended domain, particles are added by using periodic rules from particles belonging to $\Omega_{\mathrm{REV}}$. Thanks to this extension, the particles near a boundary of $\Omega_{\mathrm{REV}}$ see those from the opposite side. These additional particles are taken into account for the computation of the velocity field (Equation (6)) and for the contact modelling. The finite element computations gives the velocity for each particle inside the REV $\Omega_{\mathrm{REV}}$ by a simple interpolation. For the contact modelling, the velocity of the additional particles are deduced from those inside $\Omega_{R E V}$ by simple rules as explained in next section. 


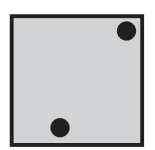

(a)

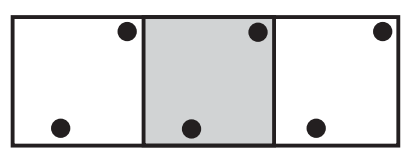

(b)

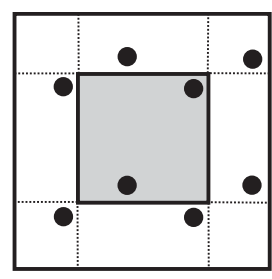

(c)

Figure 2. Extension of the computational domain $\left(\Omega_{\mathrm{REV}}\right.$ is in grey): (a) the computational domain is equal to representative elementary domain $\left(\Omega=\Omega_{\mathrm{REV}}\right)$ and $\left(k_{x}, k_{y}\right)=(0,0)$ and (b) the domain extended in the $x$-direction CL $\left(k_{x}, k_{y}\right)=(1,0)$, (c) domain with sliding biperiodic boundary conditions $\mathrm{CL}\left(k_{x}, k_{y}\right)=(1 / 2,1 / 2)$.

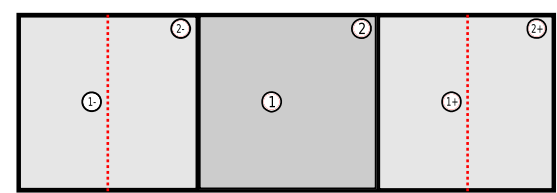

(a)

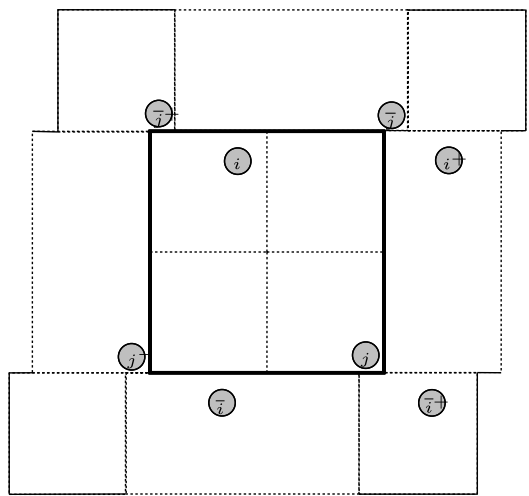

(b)

Figure 3. Schematic description of particle duplication: (a) BC with $\left(k_{x}, k_{y}\right)=(1,0)$ and (b) BC with $\left(k_{x}, k_{y}\right)=(1 / 2,1 / 2)$.

In practice, we can extend the computational domain as much as we want and $k_{x}$ and $k_{y}$ are the two real parameters in $2 \mathrm{D}$ which characterise the extension of the computational domain in, respectively, $x$ - and $y$-direction. Assuming that $\Omega_{\mathrm{REV}}$ is an unitary square, $k_{x}=1$ and $k_{y}=0$ in the configuration in Figure 2(b). Therefore, the domain is extended in $x$-direction of length 1 in both sides so that the computational domain is then three times larger than the original domain $\Omega_{\mathrm{REV}}$. In that case, if a particle is labelled by $i$, the duplicate particles $i^{+}$ and $i^{-}$which are its $x$-periodic images are obtained with the following rules in 2D,

$$
\left\{\begin{array}{l}
\text { if } x_{i}+1 \in\left[1,1+k_{x}\right], \text { then } \quad x_{i^{+}}=x_{i}+1, \\
\text { if } x_{i}-1 \in\left[1-k_{x}, 0\right], \text { then } \quad x_{i^{-}}=x_{i}-1,
\end{array}\right.
$$

their vertical components $y_{i^{ \pm}}$being unchanged. Figure 3(a) schematically represents the duplication of two particles for an extension with $\left(k_{x}, k_{y}\right)=(1,0)$.

With this modification of the boundary conditions, we decrease the influence of the vertical walls on the computation of the velocity. We can precise that only particles inside the original domain are moved. The new particles are introduced just for improving the computation of the velocity field and their positions are determined only through geometric considerations. 
In order to limit the influence of the horizontal walls, we also carry out biperiodic boundary conditions such as presented in Figure 2(c). In case of shear flows, the methodology is slightly different and has to be described more precisely. It is based on the Lees-Edwards' biperiodic boundary conditions described in Lees and Edwards, (1972) and Hwang et al. (2004). An example is presented in Figure 3(b): if $i$ and $i^{ \pm}$design a particle and its $x$-periodic images, the label $\bar{i}$ and $\bar{i}^{ \pm}$design their images by the biperiodic boundary. In order to keep the shear condition on the upper and lower boundaries of the domain of interest, it is necessary to consider sliding boundary conditions as depicted in Figure 3(b) in which the upper and lower additional domains move with velocity $\pm \gamma / 2$, respectively. Then at time $t^{n}$, the positions of the duplicated particles are given by

$$
\left\{\begin{array}{l}
\text { if } y_{i}+1 \in\left[1,1+k_{y}\right], \text { then } \quad\left(x_{\bar{i}}, y_{\bar{i}}\right)=\left(x_{i}+\frac{\dot{\gamma}}{2} t^{n}, y_{i}+1\right), \\
\text { if } y_{i}-1 \in\left[1-k_{y}, 0\right], \text { then } \quad\left(x_{\bar{i}}, y_{\bar{i}}\right)=\left(x_{i}-\frac{\dot{\gamma}}{2} t^{n}, y_{i}-1\right),
\end{array}\right.
$$

\subsection{Computation of the distances between particles}

Let us now consider the positions and the velocities of the two particles known at times $t^{n}$ and $t^{n-1}$. Using the Adams-Bashforth scheme, we are able to compute the positions of physical particles at time $t^{n+1}$ as follows:

$$
\mathbf{X}_{i}^{n+1}=\mathbf{X}_{i}^{n}+\frac{\Delta t}{2}\left(3 \mathbf{V}_{i}^{n}-\mathbf{V}_{i}^{n-1}\right)
$$

and the positions of the corresponding images are computed with the velocity of physical particles:

$$
\begin{gathered}
\mathbf{X}_{i^{ \pm}}^{n+1}=\mathbf{X}_{i^{ \pm}}^{n}+\frac{\Delta t}{2}\left(3 \mathbf{V}_{i}^{n}-\mathbf{V}_{i}^{n-1}\right) \quad \text { for the images in } x \text {-direction } \\
\mathbf{X}_{\bar{i}}^{n+1}=\mathbf{X}_{\bar{i}}^{n}+\frac{\Delta t}{2}\left(3 \mathbf{V}_{i}^{n}-\mathbf{V}_{i}^{n-1}\right) \quad \text { for the biperiodic images }
\end{gathered}
$$

For the gluey contact model, the condition that must be fulfilled in order to avoid the overlapping is the following:

$$
D=\min \left(D_{i j}, D_{i \bar{j}}, D_{i j^{ \pm}}\right) \geq 0
$$

So, we have to compute the distances between the particles. Using (14), (16) and (17), we obtain the following relationships:

$$
\begin{gathered}
D_{i j}^{n+1}=\left\|\mathbf{X}_{i} \mathbf{X}_{j}\right\|^{n}+\frac{\Delta t}{2}\left(\left(3 \mathbf{V}_{j}^{n}-\mathbf{V}_{j}^{n-1}\right)-\left(3 \mathbf{V}_{i}^{n}-\mathbf{V}_{i}^{n-1}\right)\right) \cdot \mathbf{e}_{i j} \\
D_{i j}^{n+1}=\left\|\mathbf{X}_{i} \mathbf{X}_{j}\right\|^{n}+\frac{\Delta t}{2}\left(\left(3 \mathbf{V}_{j}^{n}-\mathbf{V}_{j}^{n-1}\right)-\left(3 \mathbf{V}_{i}^{n}-\mathbf{V}_{i}^{n-1}\right) \pm j \cdot \mathbf{e}_{x}\right) \cdot \mathbf{e}_{i j} \\
D_{i j^{ \pm}}^{n+1}=\left\|\mathbf{X}_{i} \mathbf{X}_{j^{ \pm}}\right\|^{n}+\frac{\Delta t}{2}\left(\left(3 \mathbf{V}_{j}^{n}-\mathbf{V}_{j}^{n-1}\right)-\left(3 \mathbf{V}_{i}^{n}-\mathbf{V}_{i}^{n-1}\right)\right) \cdot \mathbf{e}_{i j}
\end{gathered}
$$

In the numerical procedure, the distances between physical particles and all images are computed in order to take into account the minimum value in the contact model. 


\section{Numerical rheology}

As explained in the introduction, determining rheological properties of dense suspensions remains important for many applications (Lefebvre \& Maury, 2005). For this purpose, we present here the way of calculating the macroscopic variables from our computations. Let us first note $\langle\mathbf{X}\rangle_{\Omega}$ the mean value of $\mathbf{X}$ at time $t$ over $\Omega$. We have:

$$
\langle\mathbf{X}\rangle_{\Omega}=\frac{1}{\Omega} \int_{\Omega} \mathbf{X}(\mathbf{x}) d \Omega
$$

By taking the mean value of the stress tensor $\sigma$ using (22), one gets:

$$
\langle\boldsymbol{\sigma}\rangle_{\Omega}=\left\langle\boldsymbol{\sigma}_{\mathrm{f}}\right\rangle_{\Omega}+\left\langle\boldsymbol{\sigma}_{\mathrm{s}}\right\rangle_{\Omega} \quad \text { with }\left\{\begin{array}{l}
\left\langle\boldsymbol{\sigma}_{\mathrm{s}}\right\rangle_{\Omega}=2 \eta_{\mathrm{s}}\langle\dot{\boldsymbol{\varepsilon}}(\mathbf{u})\rangle_{\Omega_{\mathrm{s}}}-\langle p\rangle_{\Omega_{\mathrm{s}}} \mathbf{I}_{\mathrm{d}}+\langle\lambda\rangle_{\Omega_{\mathrm{s}}} \\
\left\langle\boldsymbol{\sigma}_{\mathrm{f}}\right\rangle_{\Omega}=2 \eta_{\mathrm{f}}\langle\dot{\boldsymbol{\varepsilon}}(\mathbf{u})\rangle_{\Omega_{\mathrm{f}}}-\langle p\rangle_{\Omega_{\mathrm{f}}} \mathbf{I}_{\mathrm{d}}
\end{array}\right.
$$

where $\left\langle\boldsymbol{\sigma}_{\mathrm{s}}\right\rangle_{\Omega}$ and $\left\langle\boldsymbol{\sigma}_{\mathrm{f}}\right\rangle_{\Omega}$ are, respectively, the solid and fluid mean stress tensors.

Theoretically, the $x y$-component of the mean stress tensor in the suspension is given by:

$$
\left\langle\boldsymbol{\sigma}_{x y}\right\rangle_{\Omega}=2 \eta_{\mathrm{eff}}\left\langle\dot{\boldsymbol{\varepsilon}}(\mathbf{u})_{x y}\right\rangle_{\Omega_{\mathrm{f}}}
$$

which allows us to write the effective viscosity as follows:

$$
\eta_{\mathrm{eff}}=\frac{\left\langle\boldsymbol{\sigma}_{x y}\right\rangle_{\Omega}}{\langle\dot{\gamma}\rangle_{\Omega}}
$$

\section{Results}

In previous papers (Coupez et al., 2010; Verdon et al., 2010; Verdon et al., submitted), we analysed the reversibility of Stokes equations with two particles in 2D and 3D and we have studied interaction between three particles in a shear flow. These previous studies have allowed to fix some numerical parameters: a penalty factor is 10 times the fluid viscosity, eight Uzawa iterations are taken and the mesh is refined around the interface up to $10^{-3}$. In the following, we study particle motions for more important concentrations.

\subsection{Influence of the contact model}

The example presented here is the motion of 13 particles of radius $a=0.05$ in a 3D shear flow. Initially, the particles are located very close from each other (the compactness is close to 0.74 ) in order to have as many contacts as possible. Figure 4 shows the evolution of the particles trajectories in the $(x, y)$ plane up to $t=12.5 \mathrm{~s}$ (around 2 days on 16 cores).

This figure emphasises the differences induced by choice of the contact model: whereas the particles remain stuck during the whole computation with the viscous contact model, after a few time steps the cluster of particles disappears with the inelastic collisions model and the particles move away. This is due to the physical nature of the contact models. For the inelastic collisions, the value $\lambda_{i j}$ of the Lagrange multiplier can be only positive which means that it acts like a repulsive force between particles. Otherwise, in the viscous model, $\lambda_{i j}$ can be either negative or positive, which indicates it acts like a lubrication force: an attractive force is exerted on the particles when they go away from each other. 


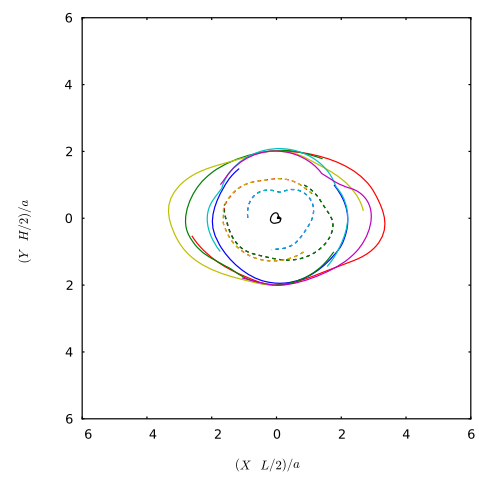

(a)

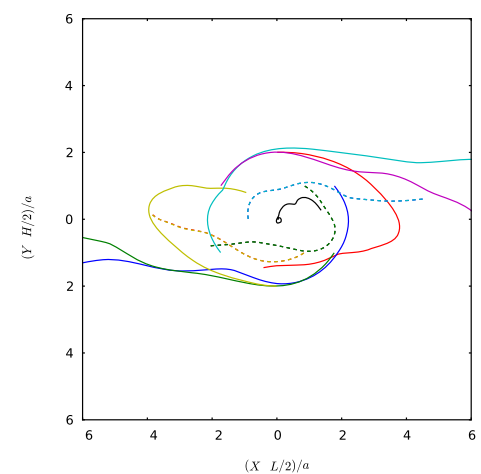

(b)

Figure 4. Influence of the contact modelling for the 13 particles. The trajectories of particles in $(x, y)$ plane: (a) viscous contact and (b) inelastic collisions.

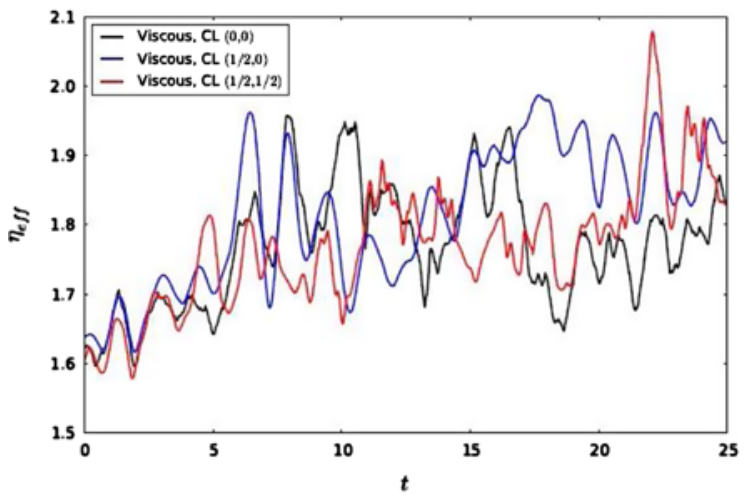

(a) Temporal evolution of $\eta_{\text {eff }}$

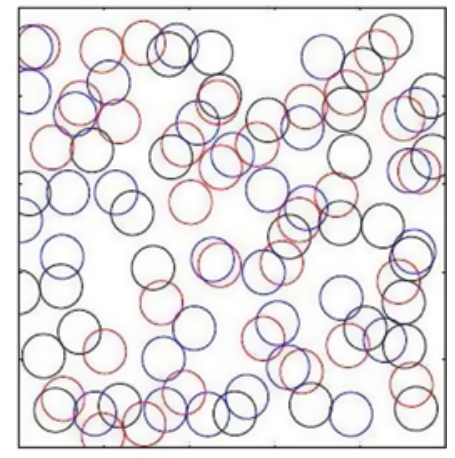

(b) Particles position after $25 \mathrm{~s}$

Figure 5. Influence of the boundary conditions for the $2 \mathrm{D}$ suspension of concentration $c \approx 0.24$.

\subsection{Influence of the boundary conditions}

In this section, we study the rheological behaviour of a $2 \mathrm{D}$ suspension of concentration $c \approx 0.24$ with computations for the three different boundary conditions. Figure 5(a) presents the time evolution of the effective viscosity whereas Figure 5(b) shows the position of the particles at $t=25 \mathrm{~s}$. Figure 5(a) shows a rather large variation of the instantaneous effective viscosity and therefore its temporal mean value $\left\langle\eta_{\mathrm{eff}}\right\rangle_{t}$ is used to look at the influence of boundary conditions. Figure 5(b) points out that boundary conditions can significantly affect the trajectories of the particles and hence the rheology of the suspension. Indeed, the biperiodic conditions limit the influence of the upper and lower walls, so that after $25 \mathrm{~s}$ the effective viscosity is lower $\left(\left\langle\eta_{\mathrm{eff}}\right\rangle_{t} \approx 1.766\right)$ than for the two other boundary conditions for which they are $\left\langle\eta_{\text {eff }}\right\rangle_{t} \approx 1.769$ and $\left\langle\eta_{\text {eff }}\right\rangle_{t} \approx 1.81$ for $\mathrm{BC}(1 / 2,0)$ and $(0,0)$, respectively. Moreover, for boundary conditions $(0,0)$, there is a minor difference between viscous and inelastic contact as the apparent viscosity is 1.778 for inelastic contact. At last, the computed values are far from empirical ones $(\sim 2.1-2.5)$, which emphasises the importance of $3 \mathrm{D}$ effects that are neglected in this study. 


\section{Conclusions}

Throughout this article, we pointed out the importance of contact modelling as well as the choice of well-suited boundary conditions. The 13 particles example shows a real big difference in the motion of the particles between the two implemented contact models whereas the 2D suspension example insists on the importance of the boundary conditions. The identification of the rheological parameters indicates that biperiodic boundary conditions limit wall effects that could be an advantage for future computations of dense suspensions. However, in our example, the influence of boundary conditions and contacts does not appear very significant. Nevertheless, we repeat here that these are just preliminary results, and in the future 3D computations for higher concentration are mandatory for understanding the real behaviour of suspensions.

\section{References}

Beaume, G. (2008). Modélisation et simulation numérique directe de l'écoulement d'un fluide complexe (Modelling and numerical simulation of a complex fluid flow) (PhD thesis, Ecole Nationale Supérieure des Mines de Paris), http://tel.archives-ouvertes.fr/tel-00416435.

Coupez, T., Digonnet, H., Hachem, E., Laure, P., Silva, L., \& Valette, R. (2010). Multidomain finite element computations: Application to multiphasic problems. In M. Souli \& D.J. Benson (Eds.), Arbitrary Lagrangian-Eulerian and fluid-structure interaction. Numerical simulation (pp. 221-289). Wiley-ISTE.

Glowinski, R., Pan, T.-W., Helsa, T., \& Joseph D. (1999). A distributed Lagrange multiplier/fictious domain method for particulate flows. International Journal of Multiphase Flows, 25, 755-794.

Hwang, W., Hulsen, M., \& Meijer, H.E.H. (2004). Direct simulation of particle suspensions in sliding bi-periodic frames. Journal of Computational Physics, 194, 742-772.

Kuhn H.W. \& Tucker A.W. (1951). Nonlinear programming. In J. Neyman (Ed.), Proceedings of the Second Berkeley Symposium on Mathematical Statistics and Probability, 31 July - 12 August 1950, University of California Press, Berkeley and Los Angeles (pp. 481-492).

Laure, P., Beaume, G., Basset, O., Silva, L., \& Coupez T. (2007). Numerical methods for solid particles in particulate flow simulations. European Journal of Computational Mechanics, 16, 365-383.

Lees, A. \& Edwards, S. (1972). The computer study of transport process under extreme condition. Journal of Physics C: Solid State Physics, 5, 1921-1928.

Lefebvre, A. (2007). Modélisation numérique d'écoulements fluide/particules (Numerical modelling of fluid/particle flows) (PhD thesis, Université Paris Sud - Paris XI, 11), http://tel.archives-ouvertes.fr/ tel-00257246/en/.

Lefebvre A. (2009). Numerical simulation of gluey particles. $M 2 A N, 43,53-80$.

Lefebvre, A. \& Maury, B. (2005). Apparent viscosity of a mixture of a Newtonian fluid and interacting particles. CRAS Mécanique, 333(12), 923-933.

Maury, B. (2007). A gluey particle model. ESAIM: Proceedings, 18, 133-142.

Patankar, N., Singh, P., Joseph, D., Glowinski, R., \& Pan, T.-W. (2000). A new formulation of the distributed Lagrange multiplier/fictitious domain method for particulate flows. International Journal of Multiphase Flow, 26, 1509-1524.

Verdon, N., Lefebvre-Lepot, A., Lobry, L., \& Laure, P. (2010). Contact problems for particles in a shear flow. European Journal of Computational Mechanics, 19, 513-531.

Verdon, N., Beaume, G., Lefebvre-Lepot, A., Lobry, L., \& Laure, P. (submitted). Influences of contact modelling in direct numerical simulations of particle suspension in a shear flow. Journal of Non Newtonian Fluid Mechanics. 